\title{
Abstracts
}

\author{
Daniel B. Lee and Achim Brosziewski
}

\section{Participant Observation and Systems Theory: Theorizing the Ground}

Ethnographers appear to be fascinated by what they immediately experience as fieldworkers. However, although they represent themselves as participant observers, they have neglected to develop a theory of observation. Systems theorists have a theory of second order observation, but they tend to avoid conducting fieldwork. In this paper, we argue that systems theory and ethnography can effectively supplement each other. Drawing on the concept of self-reference, we examine the relationship between perception, observation, and understanding. Ethnographers have not adequately explained how these fundamental concepts fit together, as we show in a discussion of Clifford Geertz' call for »thick description. « A special form of observing, understanding reaches beyond perception. Meaning and culture appear only in the light of self-reference. As participants, ethnographers must not only observe what happens in the field; but they must also observe connections between empirical events. They must observe the process of selectivity that uses meaning to differentiate what actually happens from alternative possibilities. Guided in the field by this theory of observation, ethnography can move beyond collecting »stories « and effectively open itself to the established questions, familiar problems, and epistemological resources of the discipline of sociology. For its part, a theoretically informed ethnography would help extend the scope of systems theoretical research beyond functional and historical analyses. Making their observations in the form of ethnography, systems theorists might focus on explaining how social practices unfold themselves with reference to the social dimension of meaning.

\section{Stefan Kühl}

\section{Formality, Informality and Illegality in Organizational Consulting. A System Theoretical Analysis of a Consulting Process}

If organizations are confronted with contradicting purposes they handle this contradictory needs in an informal, very often illegal way. Using system theory this article examines a consulting process in an enterprise that on the one side is promoting a market approach and on the other side has to fulfil a public mandate. The goal of the consulting process was to transfer informal solutions being developed inside the decentralized units into formal solutions. This goal could not be reached because this would have confronted the organization with contradicting needs. During the consulting process the consultants have become less and less promoters of the formalization and have been drawn into the illegality of the organization.

\section{Werner Vogd}

\section{Systems Theory: Empirical research or theoretical conceptualisation - an illu- sionary contradiction?}

Luhmann's systems theory is neither a glass bead game nor a walk across the borderline between philosophy and sociology. Its abstractions only make sense if systems are conceptualized as empirical facts. As relations these facts are neither visible nor tangible, but in principle it is possible to reconstruct them as patterns. There are only few studies that focus on the 
relationship between theory development and empirical approaches. The present paper considers the question how the system concept might be operationalized. It draws connections to qualitative and reconstructive methods, maintaining the particularity of Luhmann's concept of communication. Exemplified by the design of a research project on decision making in hospitals it will be demonstrated how empirical research might lead to a complex reconstruction that fits with Luhmann's categories as well as with polycontextural descriptions.

\section{Katharina W. Mayr}

\section{Rationality and Plausibility within HealthCare Ethics Committees Real-Time Communication as Empirical Data.}

Instead of taking up the cudgels for the Theory of Social Systems' capacity for empirical observation theoretically, this text shows with protocols of HealthCare Ethics Committees, how the analyzed data generates a world of observation that can be observed in terms of the used distinctions. The real time emergence of communicating structures will be demonstrated as a process that reduces contingency. In HealthCare Ethics Committees a new mode of communication gets visible that favours authentic subjectivity instead of organisational or medical rationality. As a new mode of reasoning emerges the participants of the HealthCare Ethics Committee are learning, that in this context the reference to organisational hierarchies or the self-confident medical action do not serve as a solution, but gets criticisable as a problem. What normally relieves of the need for reflection in everyday life of the hospital, turns into a negative pattern for the right relationship with patients and colleagues. 